\title{
The influence of working gas pressure on interlayer mixing in magnetron-deposited Mo/Si multilayers
}

\author{
Y. P. Pershyn ${ }^{* a}$, E. M. Gullikson ${ }^{\text {, I. A. Artyukov }}{ }^{\text {, }, ~ V . ~ V . ~ K o n d r a t e n k o ~}{ }^{\mathrm{a}}$, V.A. Sevryukova ${ }^{\mathrm{a}}$, \\ D. L. Voronov ${ }^{\text {, E. N. Zubarev }}{ }^{\mathrm{a}}$, A. V. Vinogradov ${ }^{\mathrm{c}}$ \\ ${ }^{a}$ National Technical University "Kharkiv Polytechnic Institute", 21 Frunze Street, \\ Kharkiv 61002, Ukraine, \\ ${ }^{\mathrm{b}}$ Lawrence Berkeley National Laboratory, Berkeley, CA 94720, USA, \\ ${ }^{c}$ P.N.Lebedev Physical Institute, 53 Leninsky Pr., Moscow, 119991, Russia
}

\begin{abstract}
Impact of Ar gas pressure (1-4 mTorr) on the growth of amorphous interlayers in Mo/Si multilayers deposited by magnetron sputtering was investigated by small-angle x-ray scattering $(\lambda=0.154 \mathrm{~nm})$ and methods of cross-sectional transmission electron microscopy. Some reduction of thickness of the amorphous inter-layers with Ar pressure increase was found, while composition of the layers was enriched with molybdenum. The interface modification resulted in raise of EUV reflectance of the Mo/Si multilayers.
\end{abstract}

Keywords: multilayer X-ray mirror, interlayer composition, Ar pressure variation, magnetron sputtering.

\section{INTRODUCTION}

Multilayer X-ray mirrors (MXMs) based on the material pair of Mo and Si are versatile in application within the wavelength range of 12.3-25.0 $\mathrm{nm}$. They are already used to serve as X-ray optical elements in microscopes ${ }^{1}$, telescopes, ${ }^{2}$ spectroscopes, ${ }^{3}$ plasma physics, ${ }^{4}$ etc. The maximal theoretical reflectivity achieves $\sim 76 \%$ at normal incidence. ${ }^{5,6}$

Measured reflectivities of Mo/Si MXMs are known to be inferior to their theoretical values by 10-15\%. As the result in a multil-mirror optical system (for example, in EUV lithographs) the total performance would worsen dramatically. The reason of the noticeable reduction of MXM reflectivity is a presence of different defects inside their structure, with interface defects such as roughness, intermixing, silicide formation playing the main role. Rough interfaces would scatter a radiation in the nonspecular direction, and intermixed and silicide interlayers decrease the gradient of optical constants (refractive indexes) thus lower the reflection amplitude at each interface and, finally, the total MXM efficiency.

As Mo-Si multilayer system is phase nonequilibrium (Mo and Si can form compounds ${ }^{7}$ ), the presence of amorphous silicide interlayers is an inherent defect of MXMs and it does not depend upon whether they are fabricated by thermal evaporation $^{8-10}$ or sputtering. ${ }^{11-13}$ The interlayer thickness at Si-on-Mo interface is in the interval $\sim 0.5-\sim 1.2 \mathrm{~nm}$ and depends on the structural state of Mo-layer: it is about $\sim 1.2 \mathrm{~nm}$ for an amorphous Mo-layer ( $a$-Mo) and usually does not exceed $\sim 0.7 \mathrm{~nm}$ if Mo-layers are crystalline (c-Mo). For Mo-on-Si interfaces the interlayer thickness makes 1.0-2.5 nm and depends on the deposition method mainly. The mechanisms of interface formation are not clear now and are the subject of the wide speculation, particularly with respect to the reason of asymmetric interfaces ${ }^{14-18}$. As for the composition, most scientists consider that the amorphous interlayers in MXMs fabricated by different methods consist of molybdenum disilicide $\left(\mathrm{MoSi}_{2}\right)^{9,}, 17,19-25$

In different studies focused on improving optical characteristic of Mo-Si multilayers the MXM reflectivity is shown to depend on the energy of deposited atoms. ${ }^{26-28}$ In particular, lowering the deposited atom energy by increasing the sputtering gas pressure results in interfaces roughening and vice versa. ${ }^{13,29,30}$ The gas pressure also influences mechanical

\footnotetext{
* persh@ kpi.kharkov.ua; phone +38 057 751-04-70
} 
stresses in multilayers. ${ }^{31,32}$ In this work we show that a variation of working gas pressure affects the width and the composition of amorphous interlayers.

\section{EXPERIMENTAL}

Multilayer mirrors were fabricated by DC magnetron sputtering. During each experimental run the discharge currents (for Si and Mo magnetrons) and Ar pressure in the vacuum chamber maintained constant, that provided the steadiness of deposition rates for both components. However when the pressure varied, the rates also changed. The range of deposition rates for molybdenum and silicon was $0.35-0.42$ and $0.45-0.65 \mathrm{~nm} / \mathrm{s}$, correspondingly, and were specially measured at every change of the pressure. Periods of MXMs were in the range from 6 to $9 \mathrm{~nm}$.

MXMs were deposited onto substrates of polished silicon wafers and float glass having RMS surface roughness of 0.3$0.5 \mathrm{~nm}$. The plates of $\mathrm{Mo}$ and $\mathrm{Si} \sim 100 \mathrm{~mm}$ in diameters with a purity of $99.5 \%$ and $99.99 \%$ correspondingly were used as targets. The distance between a magnetron surface and the substrate averages $30 \mathrm{~mm}$.

Multilayer samples were characterized at grazing angles of incidence using small-angle X-ray diffractometer DRON-3M assembled as two-crystal spectrometer with a single crystal (110)Si monochromator. In combination with a 0.1-mm slit that provided a selection of $\mathrm{CuK \alpha} \alpha_{1}$ line only from the spectrum generated by a X-ray tube with a copper anode. Phase analysis was performed in $\mathrm{Cu} K \alpha$ radiation at another diffractometer contained graphite analyzer.

Cross-sections of Mo/Si MXMs were studied in transmission electron microscope PEM-U at accelerating voltage of 100 $\mathrm{kV}$. The images were shot with a line resolution of better than $0.2 \mathrm{~nm}$ (atomic planes).

\section{RESULTS}

\subsection{Reference assumptions}

The high energy of particles is characteristic for the flow of matter originated from a sputtering source. This results in a formation of intermixed interlayers in Mo/Si MXMs. We tried to reduce this effect at the expense of decreasing the energy of particles impinging a growing surface by changing the gas pressure inside the vacuum chamber. When a mean free path for sputtered atoms becomes comparable with the distance from the magnetron till the substrate, the energy of atoms should fall down, and further increase of the pressure must eventually bring to a thermalization of sputtered atoms. Therefore to manipulate the energy of deposited particles we fabricated a series of multilayer samples with sequential change in pressure of sputtering gas Ar ranging from 1 till 4 mTorr. The mean free path in doing so varied from $\sim 53 \mathrm{~mm}$ down to $\sim 13 \mathrm{~mm}$.

The composition of amorphous interlayers in Mo/Si MXMs has a silicide character. So formation of each interlayers is accompanied by volume contraction since the total volume of parent components involved in the reaction is larger than the volume of reacted products. Maximal contraction is expected when $\mathrm{MoSi}_{2}$ is formed, and for tabulated densities it reaches up to $\sim 27.2 \%$. This contraction should decrease the MXM periodicity against the expected one. We used this fact to evaluate the degree of layer interaction. Thus a difference, $\Delta d$, between expected MXM period, $d_{0}$, and experimental one, $d_{M}$, is taken as a measure of intermixing and interaction in Mo-Si multilayer system.

If deposition rates and times are known the values of the expected periods can be estimated by the following formula:

$$
d_{0}=V_{M o} \times \tau_{M o}+V_{S i} \times \tau_{S i},
$$

where: $\quad V_{M o}$ and $V_{S i}-$ deposition rates for molybdenum and silicon layers, correspondingly;

$\tau_{M o}$ and $\tau_{S i}-$ deposition times for Mo and Si layers, correspondingly.

To measure the real deposition rates of components we fabricated MXMs consisting of three periodic stacks differing by the deposition time of each component. In two sequentially deposited stacks therewith we changed the deposition time for only one component while the time for the other was the same. The difference in periods for adjacent stacks divided by the difference in times gave the deposition rate of the given component. 
The period of each deposited stack was determined on the base of full Bragg equation adjusted for the refraction employing the least-squares method. The period value in this case can be determined with a precision of better than 0.01 $\mathrm{nm}$.

It should be also noted that two multilayer samples were fabricated in each experiment (labeled as $\boldsymbol{A}$ and $\boldsymbol{B}$ ) which were fixed on the rotating substrate holder diametrally opposite. That was made to raise an authenticity of the findings. The multilayer construction for the $\boldsymbol{A}$ and $\boldsymbol{B}$ samples is asymmetric as against each other with a view to an order of the thickness change for each component, i.e. changing the thickness for one component at the sample $\boldsymbol{A}$ was automatically attended by changing the thickness of another component at the sample $\boldsymbol{B}$. That is why two stacks of the sample $\boldsymbol{A}$ consisted of thinner Mo layers while there are two stacks of thicker Mo layers in the sample $\boldsymbol{B}$. The similar situation occurred for Si layers but in a contrary way.

\subsection{Small-angle measurements at hard $X$-rays $(\lambda=0.154 \mathrm{~nm})$}

Results on measuring the deposition rates for Mo and Si in the range of sputtering pressures of 1-4 mTorr are presented in Fig. 1. As it can be seen the rates for corresponding components of $\boldsymbol{A}$ and $\boldsymbol{B}$ series are close. Their small variations are mainly connected with a slight difference in geometrical configuration during the process of the deposition in the positions $\boldsymbol{A}$ and $\boldsymbol{B}$. Generally deposition rates for both series progressively fall down as the pressure increases, and that is related to decaying the glow-discharge potential for each magnetron. The sole exception is the region of large pressures: at $\sim 3$ mTorr a growth of deposition rates is observed. This fact is unexpected since it contradicts the data of the sputtering parameter control. It is known that the deposition rate of sputtered matter varies directly as the electric power consumed by a magnetron. ${ }^{33}$ At the same time we observed only a decay of the requisite power for each magnetron when the Ar pressure increases. In other words the observed "growth" of deposition rates is the property of the studied object itself rather than a peculiarity of changing the parameters of glow discharge at magnetrons. We will return back to the discussion of possible reason for this feature later.

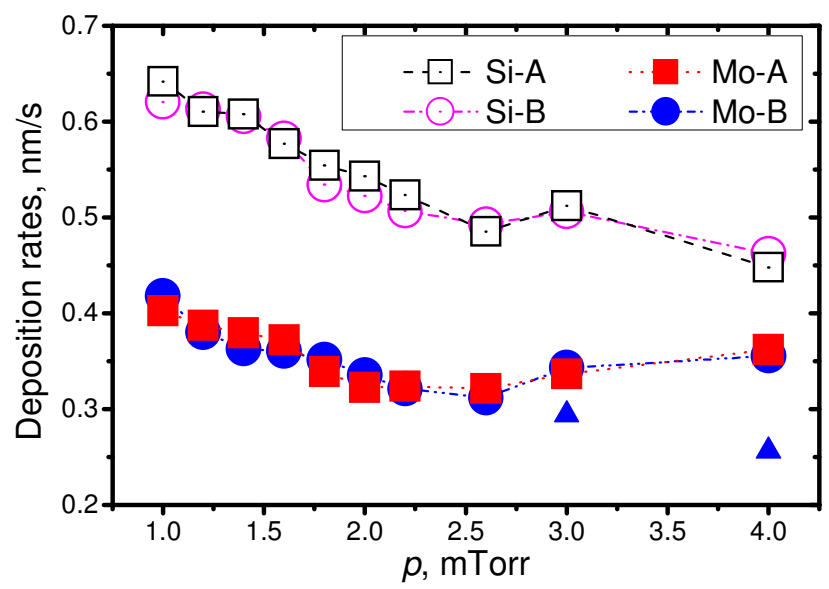

Figure 1. The deposition rates for Mo and Si of A and B series versus the pressure of sputtering gas Ar.

Now knowing all constituents in formula (1), we determine expected values of periods $\left(d_{O}\right)$ and find the difference $(\Delta d)$ between expected and measured $\left(d_{M}\right)$ periods, i.e. the MXM contraction. We attribute that difference directly to the interlayer interaction in Mo-Si system under changes of the working gas pressure. Processed data for all experiments are combined in Fig. 2. As illustrated, the contraction with the pressure does not vary monotonically: at low pressures (1.01.6 mTorr $)$ it is relatively unaffected $(0.36-0.49 \mathrm{~nm})$ and then drops severely at least by factor of $\sim 5$ down to $0.02-0.08$ $\mathrm{nm}$ at 2.0-2.6 mTorr. Such fall in $\Delta d$ can be an evidence of a noticeable decline in intermixing the Mo-Si multilayer system. The boundary pressure, wherein such drop begins, is $\sim 1.8$ mTorr. At this pressure the mean free path is comparable with the distance between a magnetron and a substrate. At 3 mTorr and above the contractions ascend significantly again, and their values exceed ones for low pressures ( $p<1.6 \mathrm{~m}$ Torr) at that. This peculiarity is observed for the same pressures as the deviation in deposition rates (Fig. 1). 
We suppose that the contraction growth at Ar pressures $\mathrm{p} \geq 3 \mathrm{mTorr}$ can be caused by the following reason. As it was ascertained hitherto molybdenum in Mo/Si multilayered samples can be in two states: crystalline or amorphous. ${ }^{23}$ The transition from amorphous to crystalline state was seen in the range of thicknesses $t_{\mathrm{Mo}_{\mathrm{o}}} \sim 2.0-2.6 \mathrm{~nm} .^{24,25}$ The silicide thicknesses differ substantially for these cases. When MXMs are deposited by DC magnetron the silicide interlayers around $a$-Mo have the identical thickness of $\sim 1 \mathrm{~nm}$. Interlayers at adjacent interfaces of $c$-Mo are distinguished by thickness: ${ }^{23,24}$ 0.9-1.1 $\mathrm{nm}$ at Mo-on Si interfaces and 0.4-0.6 nm at Si-on-Mo interfaces. Total thickness of interfaces for both cases differs by 0.3-0.7 nm. In other words the amorphization of Mo-layers should result in a rise of the period contraction.

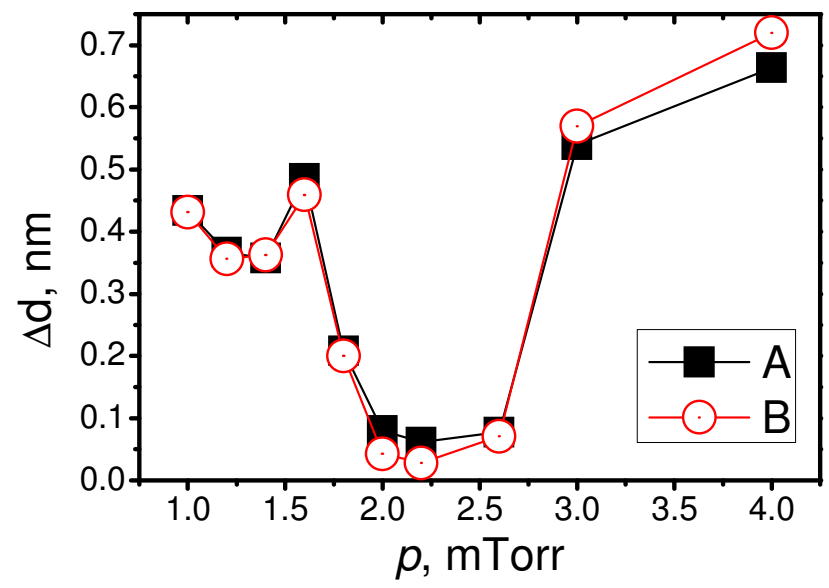

Figure 2. The dependence of the period contraction for Mo/Si MXMs versus the pressure of working gas Ar.

Evaluated by the measured deposition rates the thickness of thinnest Mo-layers for Ar pressures of 3 and 4 mTorr comprises 2.8-2.9 nm, that is outside the limits of the amorphous-crystalline transition. However we also made an estimate of Mo deposition rates supposing that they vary directly as the applied power and found that the real thickness can be less taking values of 2.1-2.4 nm (triangles in Fig. 1), that is within the amorphization range. Since each sample has multilayer stacks with two distinct thicknesses of molybdenum, then visible growth of Mo deposition rates must be connected with the difference in the structural states of thin and relatively thick Mo-layers at the boundary of the amorphous-crystal transition.

As stated above $\mathrm{MoSi}_{2}$ is considered as the main silicide to be formed at interfaces during the sputter deposition. ${ }^{9,19,20,22,24}$ With the formal approach to the description of the disilicide reaction $\left(\mathrm{Mo}+\mathrm{Si} \rightarrow \mathrm{MoSi}_{2}\right)$ and tabulated densities of components $\left(\rho_{\mathrm{Si}}=2.332 \mathrm{~g} / \mathrm{cm}^{3}, \rho_{\mathrm{Mo}}=10.218 \mathrm{~g} / \mathrm{cm}^{3}, \rho_{\mathrm{MoSi} 2}=6.24 \mathrm{~g} / \mathrm{cm}^{3}\right)$, we calculated volume ratios of components and the expected contraction to establish a relationship between silicide volume and corresponding contraction coming out to $V_{M o S i 2} / \Delta V=2.68$. Assuming that the reaction of silicide formation goes normally to interfaces only, this equation can be rewritten as $t_{M o S i 2}=2.68 \times \Delta d$. Results of the estimation for total silicide thickness of two adjacent Mo-Si interfaces are shown in Fig. 3. Here we also added published values of $t_{\mathrm{MoSi}}$ taken from cross-sections of Mo/Si MXMs in TEM (patterned rectangles).

As expected, the Ar pressure dependence of silicide thickness repeats the $\Delta d(p)$ plot in Fig. 2 but on a larger scale. Interlayer thicknesses obtained in this study are slightly thinner than literature values (patterned regions). Small tilt of the samples in the TEM column and interfaces roughness may be responsible for excessive values of the interface thickness. Besides the density of formed silicide can be lower than tabulated one, that exists with regards to thin films, ${ }^{34,35}$ and actual silicide thickness may be larger.

\subsection{Phase composition of Mo/Si MXMs}

As far as the nonmonotonic decay for the deposition rate may be associated with the amorphous-crystalline transition for Mo-layers, we analyzed the structural state of Mo depending on the gas pressure. The examination of large-angle diffraction curves revealed that Mo-layers are crystalline (Fig. 4). Any reflexes corresponding to silicon or silicide interlayers with the exception of a wide halo at $2 \theta \sim 28^{\circ}$ are absent, that suggests their amorphous structure. Diffraction patterns for samples of $\boldsymbol{A}$ and $\boldsymbol{B}$ series except for those made at 4 mTorr are almost identical to each other. That is fair 
indication that at least in the pressure range of 1.0-2.6 mTorr where the pronounced drop of the contraction is observed (Fig. 2) there is no any changes in the structural state of Mo-layers.

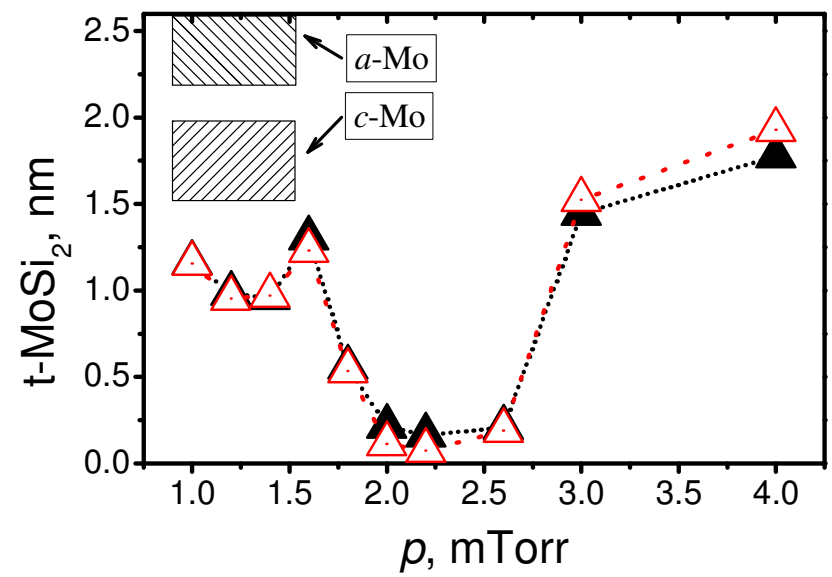

Figure 3. The estimates of silicide layer thickness $\left(\mathrm{MoSi}_{2}\right)$ in $\mathrm{Mo} / \mathrm{Si} \mathrm{MXMs}$ as a function of the pressure of sputtering gas Ar. Patterned regions show silicide thickness given in the literature for amorphous ( $a$-Mo) and crystalline $(c$-Mo) molybdenum layers.

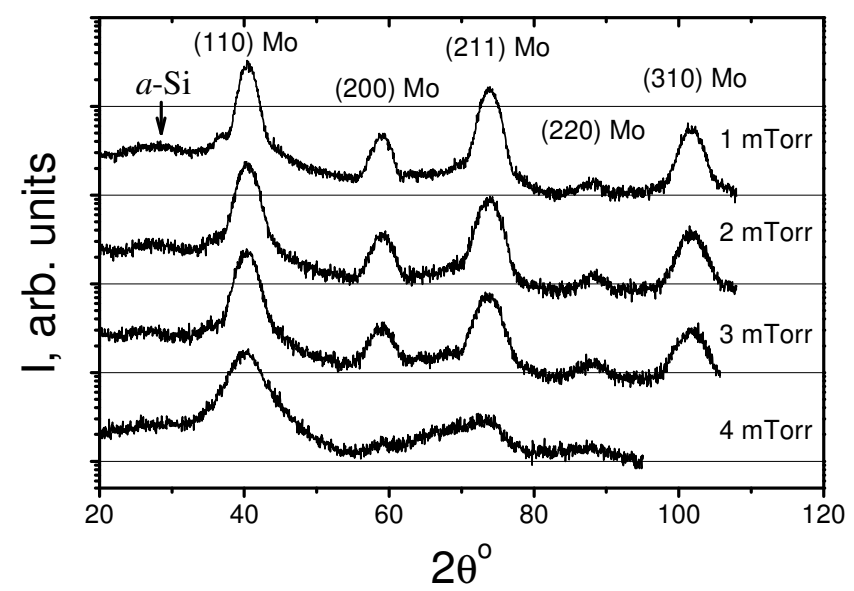

Figure 4. Hard X-ray diffraction curves $(\lambda=0.154 \mathrm{~nm})$ for Mo/Si MXMs showing the change of the structural state with the change of Ar pressure. Curves are shifted by order of the magnitude apart for convenience.

Also present in the diffraction patterns is a sequential shift in the position of (110) Mo peaks from $2 \theta \sim 40.5^{\circ}$ ( 1 mTorr) to $\sim 40.2^{\circ}$ (4 mTorr), i. e. interplanar spacings for (110)Mo grow with pressure (Fig. 5). The Mo-layers deposited by magnetron sputtering at low Ar pressures ( 1 mTorr) are described ${ }^{36}$ to be positively stressed. When the pressure increases the inversion of stresses is observed. The change of sign in stresses occurs around 2 mTorr. Such changeover must be accompanied by decreasing the interplanar spacing for the atomic planes being parallel or at a small angle to the film plane at the expense of a lateral contraction and corresponding shift of diffraction peaks to larger angles. As we can see the opposite situation occurs in our case. In other words the observed shift of peak position is not concerned with stresses.

In our earlier study ${ }^{37}$ on MXMs with $a-\mathrm{Mo}\left(\mathrm{t}_{\mathrm{Mo}}<2 \mathrm{~nm}\right)$ we found that the angle position of most intense peak is located at $2 \theta \sim 39.9^{\circ}$ while the tabulated position for (110)Mo must be at $40.49^{\circ}$ with $\mathrm{Cu} K \alpha$ radiation. The displacement of the angle position for (110)Mo by $\sim 0.3^{\circ}$ with increasing pressure implies a partial amorphization of Mo-layers deposited at elevated pressures ( $p \geq 3$ mTorr). 
In addition to that a noticeable deterioration of the structural perfection of Mo crystal structure occurs at 4 mTorr manifested in a disproportionate lowering of intensities for other diffraction peaks (Fig. 4). For the samples of $\boldsymbol{A}$ and $\boldsymbol{B}$ series with predominantly thin and thick Mo-layers accordingly the intensities of (110)Mo peaks differ by factor of $\sim 2$. All these data point to the possibility of existing $a$-Mo in thin layers of the samples deposited at 3 and 4 mTorr.

Thus, the amorphization of thin Mo films brings about the overestimation of deposition rates for components at the boundary of the amorphous-crystalline transition for Mo-layers deposited at Ar pressures of $\mathrm{p} \geq 3 \mathrm{mTorr}$. In this situation the effect of the contraction drop for MXMs is distorted.

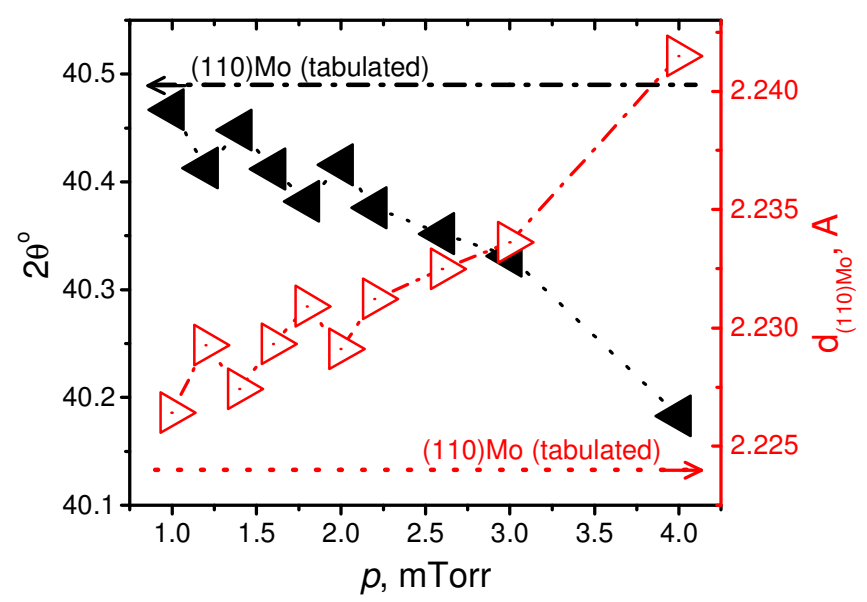

Figure 5. The shift of (110)Mo angle position (full triangles) and corresponding interplanar distances (open triangles) in Mo/Si MXMs as a function of Ar pressure.

\subsection{Electron-microscopy study}

Separately we prepared multilayer samples on silicon wafers at Ar pressure of 1.4, 2 and 4 mTorr for cross-sectional TEM study. MXM periods were $\sim 11 \mathrm{~nm}$. Such relatively large periods were chosen to avoid an appearance of $a$-Mo layers and study MXMs with $c$-Mo layers only.

In TEM images the crystalline grains are revealed within the Mo-layers at the expense of the diffraction contrast. The grains are single-block and their height conforms to the Mo-layer thickness. Their dimensions sideward are many times longer and reach $\sim 30 \mathrm{~nm}$. So Mo-layer are in the crystalline form only. Silicon layers are amorphous and separated from Mo-layers by amorphous interlayers. According to selected-area electron diffraction patterns the Mo-grains are textured for all samples. The texture axis [110] is perpendicular to the MXM layers.

Interlayer thickness at Mo-on-Si interfaces for the sample deposited at $1.4 \mathrm{mTorr}$ is 1.2-1.4 nm. At adjacent interfaces (Si-on-Mo ones) the interlayers are thinner by factor of $\sim 1.5$. The rise of Ar pressure to $2 \mathrm{mTorr}$ does not involve any substantial changes in the structural state. Insignificant reduction by $\sim 0.2 \mathrm{~nm}$ for interlayer thickness was noted but this value is comparable with the TEM resolution. Further growth of the pressure till 4 mTorr is accompanied by an appreciable development of the interface roughness being on the rise from the substrate to the surface and a deterioration of the axial texture perfection. Meanwhile the visible thickness of interlayers at bottom Mo-interface decreases down to $\sim 1 \mathrm{~nm}$.

\subsection{EUV measurements (13.2-13.6 nm)}

To measure the EUV reflectivity we prepared single-period Mo/Si MXMs $(\mathrm{d} \sim 7 \mathrm{~nm})$ at Ar pressures ranging from 1.4 till 2.6 mTorr. That pressure range includes the critical point ( 1.8 mTorr) wherein the slump of MXM contraction is observed (see subsection 3.2). MXM characteristics and results of measurements are listed in Table 1 and illustrated in Fig. 6. It is visible from the table that reflectivities in hard X-ray and EUV ranges do not correlate each other. 
Table 1. Parameters of Mo/Si multilayer X-ray mirrors and results of measuring the reflectivities in EUV ranges at normal incidence.

\begin{tabular}{|c|c|c|c|c|c|c|}
\hline No. & $\begin{array}{c}\mathbf{p}_{\text {Ar }}, \\
\mathbf{m} \text { Torr }\end{array}$ & $\begin{array}{c}\mathbf{d}, \\
\mathbf{n m}\end{array}$ & $\begin{array}{c}\mathbf{R}_{\mathbf{1}}, \boldsymbol{\%} \\
(\mathbf{0 . 1 5 4} \mathbf{~ n m})\end{array}$ & $\begin{array}{c}\mathbf{R}, \boldsymbol{\%} \\
\text { (normal incidence) }\end{array}$ & $\boldsymbol{\lambda}, \mathbf{n m}$ & $\boldsymbol{\Delta} \boldsymbol{\lambda}, \mathbf{n m}$ \\
\hline 1 & 1.4 & 6.87 & 73.6 & 54.6 & 13.4 & 0.51 \\
\hline 2 & 1.8 & 6.91 & 72.0 & 59.9 & 13.4 & 0.51 \\
\hline 3 & 2.0 & 6.95 & 72.6 & 61.3 & 13.5 & 0.52 \\
\hline 4 & 2.6 & 6.84 & 71.1 & 59.9 & 13.3 & 0.49 \\
\hline
\end{tabular}

The pressure dependence of EUV reflectivity is a nonmonotonic function (Fig. 6). There is a maximum around 2.2 mTorr. Reflectivity growth at low pressures $(1.4<p<2.2$ mTorr) signifies that a refractive index gradient at Mo-Si interfaces increases, i.e. the activity of silicide formation at interfaces decays. After $2.4 \mathrm{mTorr}$ the reflectivity goes down. Such reduction must be connected with the development of the interface roughness at higher pressure, that is displayed in TEM of this study (see subsection 3.4) and in studies of other authors. ${ }^{26,38}$ The existence of maximum alludes that there is a trade-off between a rise of optical contrast and the roughness development.

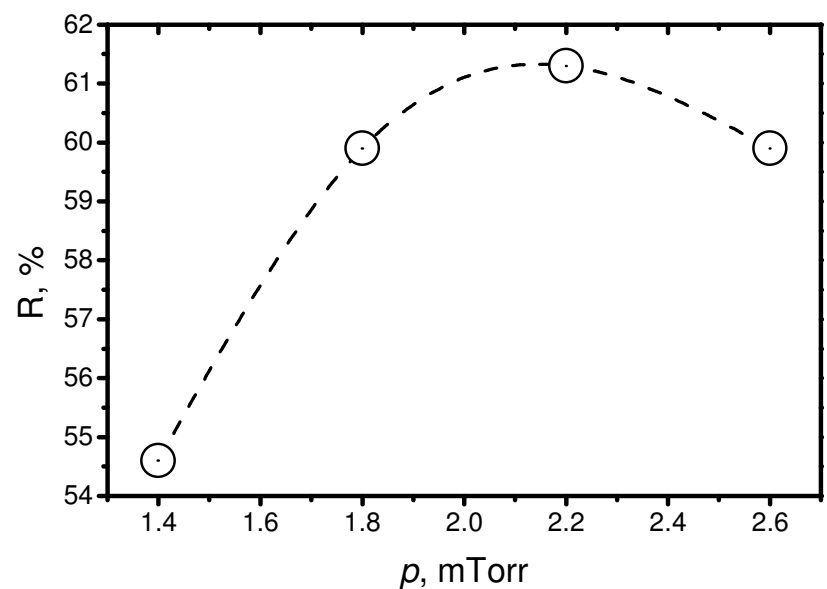

Figure 6. The dependence of the reflectivity at normal incidence for Mo/Si MXMs in EUV region $(\lambda=13.2-13.6 \mathrm{~nm})$ as function of Ar pressure. The dotted line is given just as a guide for the eyes.

\section{DISCUSSION}

As it was shown in subsection 3.4, there is a weak tendency for the interlayer thickness to decrease when the pressure increases, in spite of the fact that estimated contractions demonstrate their significant shortening (see subsection 3.2). The total thickness of $\mathrm{MoSi}_{2}$ interlayers, according to our estimates, is no more than $0.21 \mathrm{~nm}$ at moderate pressures $(1.8<p<3.0 \mathrm{mTorr})$. At the same time the interlayers for Mo-on-Si interfaces in TEM images are at least $1 \mathrm{~nm}$ thick, i.e. about five time larger. One of possible reasons capable to resolve this contradiction is a modification of the interface composition with pressure variation.

There are experiment data for e-beam MXMs authenticating a formation of interlayers with a composition of Moenriched silicides ${ }^{39}$ for example $\mathrm{Mo}_{5} \mathrm{Si}_{3}{ }^{40,41}$ and $\mathrm{Mo}_{3} \mathrm{Si}^{41}$ It was also shown that the interlayer composition is changed from $\mathrm{Mo}_{5} \mathrm{Si}_{3}$ to $\mathrm{MoSi}_{2}$ depending on the energy of $\mathrm{Kr}+$ ions used for smoothing Si-layer surface. ${ }^{40}$ The energy of atoms deposited from the thermal sources is low $(0.1-0.2 \mathrm{eV})$ that is why the formation of lower silicides could be expected for such deposition methods. However it was shown by Maury et al. ${ }^{42}$ that in sputter-deposited Mo/Si MXM (the energy of Mo-atoms is at least one order of magnitude higher compared to that for thermal evaporation) the $\mathrm{Mo}_{5} \mathrm{Si}_{3}$ silicide is formed at Mo-on-Si interfaces. From the data reported in the literature it is possible to trace a tendency of appearing Sienriched silicides as the energy released at interfaces increases. It is also confirmed by the fact of growing the amorphous interlayers with a composition of $\mathrm{MoSi}_{\mathrm{x}}(\mathrm{x} \sim 3.9)$ in the process of irradiating $\mathrm{Mo} / \mathrm{Si} \mathrm{MXMs}$ with $\mathrm{Ar}+$ ions having the energy of $\sim 180 \mathrm{keV} .^{43}$ 
Having made the estimations similar to that in the subsection 3.2, we found that volume ratios between a silicide and the corresponding contraction came for $\mathrm{Mo}_{5} \mathrm{Si}_{3}$ to $\mathrm{V}_{\mathrm{Mo5Si}} / \Delta \mathrm{V} \sim 4.8\left(\rho_{\mathrm{Mo5Si3}}=8.24 \mathrm{~g} / \mathrm{cm}^{3}\right)$ and for $\mathrm{Mo}_{3} \mathrm{Si}_{\text {to }} \mathrm{V}_{\mathrm{Mo3si}} / \Delta \mathrm{V} \sim 7.1$ $\left(\rho_{\text {Mo3Si }}=8.97 \mathrm{~g} / \mathrm{cm}^{3}\right)$. Then minimal contraction of 0.07-0.21 nm (Fig. 2) must give rise of $\mathrm{Mo}_{5} \mathrm{Si}_{3}$ 0.34-1.01 nm thick or $\mathrm{Mo}_{3} \mathrm{Si}$ 0.5-1.49 nm thick. We correlated our estimations with the TEM data (the subsection 3.4) and drew a conclusion that one of interfaces in Mo/Si MXMs deposited at Ar pressures $p>1.8$ mTorr has a composition close to $\mathrm{Mo}_{5} \mathrm{Si}_{3}$ or silicide mixture of $\mathrm{Mo}_{5} \mathrm{Si}_{3}$ and $\mathrm{Mo}_{3} \mathrm{Si}$.

The reflectivities of magnetron-deposited Mo/Si MXMs are usually $65-68 \%$ in this EUV wavelength range. ${ }^{16}$ Such modest reflectivities of fabricated MXMs can be attributed to different reasons, for instance: high interface roughness, wide interfaces, nonoptimal ratio of layer thickness, surface and volume pollutions etc. We considered one after another the influence of each item fitting a simulating curve to the experimental one in EUV range for the sample deposited at 1.4 mTorr.

It is worth to note here that each of listed defects is capable to reduce the MXM reflectivity down to $54.6 \%$ ( $\lambda=13.4 \mathrm{~nm}$ ) but adjustable parameters takes extremely high values. For example, the interface roughness, $\sigma$, must be more than $1 \mathrm{~nm}$, or the fraction, $\beta$, of Mo-containing layer must be more than 0.85, or the MXM should not have the layer of pure molybdenum, that is in contradiction to the results of the fit in hard X-ray region $\left(\sigma<0.45 \mathrm{~nm}, \beta \sim 0.55, \mathrm{t}_{c-\mathrm{Mo}_{0}} \sim 2.5 \mathrm{~nm}\right)$. If impurities are the main reason of the reflectivity lowering then their content should amount up to tens of percent $(>20 \%$ for oxygen or $>30 \%$ for carbon) that is also in conflict with our indirect measurements. It would be possible to assume that each parameter may give its small contribution in general reflectivity decrease so that their total influence will be essential. However aside from negative effect on R, each parameter causes narrowing FWHM of the reflecting curve at least by $10 \%$ and makes a full-profile analysis impossible.

One of the parameters affecting the reflectivity without noticeable change in peak width is an absorbing surface layer that can be formed at the expense of top Mo-layer oxidizing ${ }^{44}$ or post-deposition covering the MXM with a thin polluting film. We terminated the process of MXM fabrication by depositing Si-layer $\sim 4.5 \mathrm{~nm}$ thick. Taking into account that $\sim 1$ $\mathrm{nm}$ of $\mathrm{Si}$ was spent to form the silicide interlayer and $\sim 1.5 \mathrm{~nm}$ to form top oxide layer, the rest $\sim 2.5 \mathrm{~nm}$ of Si must surely protect top Mo-layer from the oxidation. Additionally we also made an analysis of small-angle reflecting curve $(\lambda=0.154$ $\mathrm{nm}$ ) for the bare substrate stored for several months in the conditions similar to those for studied samples and revealed an oscillating curve from a coating that was absent on a fresh substrate. The fitting curve allowed to establish that the coating (probably hydrocarbon one) was 6-7 $\mathrm{nm}$ thick with a density of $\sim 2 \mathrm{~g} / \mathrm{cm}^{3}$. Such coating could decrease the EUV reflectivity at least by $8 \%$, that is a half of the total reflectivity decrease.

\section{CONCLUSIONS}

It is experimentally demonstrated the fact of decreasing the period contraction and amorphous interlayer thickness in $\mathrm{Mo} / \mathrm{Si}$ MXMs deposited by magnetron sputtering when working Ar gas increases from 1 to 4 mTorr. This is followed by a change of the interlayer composition from $\mathrm{MoSi}_{2}$ to $\mathrm{Mo}_{5} \mathrm{Si}_{3}$ or the mixture of $\mathrm{Mo}_{5} \mathrm{Si}_{3}$ and $\mathrm{Mo}_{3} \mathrm{Si}$ and corresponding response in the volume shrinkage. The critical pressure giving the maximal drop in interlayer thickness is that under which the mean free path for the atoms of Ar working gas is less than the distance between the magnetron and the substrate. The composition change and thinning the interlayer thickness is connected with decreasing the energy of atoms deposited onto the growing surface at the expense of collisions with working gas atoms and a transfer them an excessive energy.

The interlayer composition change-over is attended by the growth of Mo/Si MXM reflectivity in EUV region at least by $5 \%$.

A new study is now in progress to optimize the construction of Mo/Si MXMs and the deposition technology.

\section{ACKNOWLEDGEMENTS}

The Advanced Light Source is supported by the Director, Office of Science, Office of Basic Energy Sciences, Material Science Division, of the U.S. Department of Energy under Contract No. DE-AC02-05CH11231 at Lawrence Berkeley National Laboratory. 


\section{DISCLAIMER}

This document was prepared as an account of work sponsored by the United States Government. While this document is believed to contain correct information, neither the United States Government nor any agency thereof, nor The Regents of the University of California, nor any of their employees, makes any warranty, express or implied, or assumes any legal responsibility for the accuracy, completeness, or usefulness of any information, apparatus, product, or process disclosed, or represents that its use would not infringe privately owned rights. Reference herein to any specific commercial product, process, or service by its trade name, trademark, manufacturer, or otherwise, does not necessarily constitute or imply its endorsement, recommendation, or favoring by the United States Government or any agency thereof, or The Regents of the University of California. The views and opinions of authors expressed herein do not necessarily state or reflect those of the United States Government or any agency thereof or The Regents of the University of California.

\section{REFERENCES}

[1] Artyukov, I. A., Fedorenko, A. I., Kondratenko, V. V., Yulin, S. A. and Vinogradov, A. V., "Soft X-ray submicron imaging experiments with nanosecond exposure," Opt. Commun. 102, 401-406 (1993).

[2] Golub, L., Herant, M., Kalata, K., Lovas, I., Nystrom, G., Pardo, F., Spiller, E. and Wilczynski, J., "Sub-arcsecond observations of the solar X-ray corona," Nature 344, 842-844 (1990).

[3] Levashov, V. E., Zubarev, E. N., Fedorenko, A. I., Kondratenko, V. V., Poltseva, O. V., Yulin, S. A., Struk, I. I. and Vinogradov, A. V., "High throughput and resolution compact spectrograph for the 124-250 ̊ range based on $\mathrm{MoSi}_{2}-\mathrm{Si}$ sliced multilayer grating," Opt. Commun. 109, 1-4 (1994).

[4] Bowering, N. R., Hoffman, J. R., Khodykin, O. V., Rettig, C. L., Hansson, B. A. M., Ershov, A. I. and Fomenkov, I. V., "Metrology of laser-produced plasma light source for EUV lithography," Proc. of SPIE 5752, 1248-1256 (2005).

[5] Yakshin, A.E., van de Kruijs, R.W.E., Nedelcu, I., Zoethout, E., Louis, E., Bijkerk, F., Enkisch, H. and Mullender, S., "Enhanced reflectance of interface engineered Mo/Si multilayers produced by thermal particle deposition," Proc. of SPIE 6517, 65170I (2007).

[6] Braun, S., Mai, H., Moss, M., Scholz, R. and Leson, A., "Mo/Si multilayers with different barrier layers for applications as extreme ultraviolet mirrors," Jap. J. Appl. Phys. 41, 4074-4081 (2002).

[7] Lyakishev, N. P. (Ed.), [Handbook on Binary State Diagrams of Metallic Systems], Mashinostroenie, Moscow (1996) (in Russian).

[8] Stearns, M. B., Chang, C.-H. and Stearns, D. G., "Optimization of growth conditions of vapor deposited Mo/Si multilayers," J. Appl. Phys. 71, 187-195 (1992).

[9] Braun, S., Dietsch, R., Haidl, M., Holz, Th., Mai, H., Mullender, S. and Scholz, R., "Mo/Si-multilayers for EUV applications prepared by Pulsed Laser Deposition (PLD)," Microelectron. Eng. 57-58, 9-15 (2001).

[10] Nedelcu, I., van de Kruijs, R. W. E., Yakshin, A. E., Tichelaar, F., Zoethout, E., Louis, E., Enkisch, H., Muellender, S., and Bijkerk, F., "Interface roughness in Mo/Si multilayers," Thin Solid Films 515, 434-438 (2006).

[11] Petford-Long,, A. K., Stearns, M. B., C.-H. Chang, S. R. Nutt, Stearns, D. G., Cegiio, N. M. and Hawryluk, A. M., "High-resolution electron microscopy study of x-ray multilayer structures," J. Appl. Phys. 61, 1422-1428 (1987).

[12] Holloway, K., Do, K. B. and Sinclair, R., "Interfacial reactions on annealing molybdenum-silicon multilayers," J. Appl. Phys. 65, 474-480 (1989).

[13] Steams, D. G., Rosen, R. S. and Vernon, S. P., "High-performance multilayer mirrors for soft x-ray projection lithography," Proc. SPIE 1547, 2-13 (1991).

[14]Zubarev, E. N., Kondratenko, V. V., Poltseva, O. V., Sevryukova, V. A., Fedorenko, A. I., and Yulin, S. A., "Interface intermixed zones in Mo-Si multilayers," Metallofizika i noveishie tehnologii 19, 56-63 (1997) (in Russian).

[15] Yulin, S., Feigl, T., Kuhlmann, T., Kaiser, N., Fedorenko, A. I. , Kondratenko, V. V., Poltseva, O. V., Sevryukova, V. A., Zolotaryov, A. Yu. and Zubarev, E. N., "Interlayer transition zones in Mo/Si superlattices," J. Appl. Phys. 92, 1216-1220 (2002).

[16] Largeron, C., Quesnel, E. and Thibault, J., "Interface growth mechanism in ion beam sputtering-deposited Mo/Si multilayers," Phil. Mag. 86, 2865-2879 (2006). 
[17] Wu, L., Wei, S., Wang, B. and Liu, W., "Interlayer microstructure of sputtered Mo/Si multilayers," J. Phys.: Condens. Matter 9, 3521-3528 (1997).

[18] Morgan, W. L. and Boercker, D. B., "Simulating growth of Mo/Si multilayers," Appl. Phys. Lett. 59(10), 1176-1179 (1991).

[19] Stearns, D. G., Rosen, R. S. and Vernon, S. P., "High-performance multilayer mirrors for soft x-ray projection lithography," Proc. SPIE 1547, 2-13 (1991).

[20] Boher, P., Houdy, Ph., Hennet, L., Küline, M., Müller, P., Frontier, J. P., Trouslard, P. ,Senillou, C., Joud, J. C. , Ruterana, P., "Structural characteristics and performances of rf-sputtered $\mathrm{Mo} / \mathrm{Si}$ and $\mathrm{Co} / \mathrm{Si}$ multilayers for soft X-ray optics," Proc. SPIE 1547, 21-38 (1991).

[21] Mayerheim, H. L., Döbler, U., Puschmann, A. and Babersshke, K., "Amorphous molybdenum silicide layers and $\mathrm{Mo} / \mathrm{Si}(100)$ interface growth: Local structure and preparation dependence," Phys. Rev. B 41, 5871-5880 (1990).

[22] Slaughter, J. M., Shapiro, Arye, Kearney, Patrick A. and Falco, Charles M., "Growth of molybdenum on silicon: Structure and interface formation," Phys. Rev. B44, 3854-3863 (1991).

[23]Zubarev, E. N., Kondratenko, V. V., Sevryukova, V. A., Yulin, S. A., Feigl, T., Kuhlmann, T. and Kaiser, N., "Influence of the structural state for molybdenum layers on the formation of interlayer transition zones in $\mathrm{Mo} / \mathrm{Si}$ multilayer composotions," Metallofizika I noveishie tehnologii 24(10), 1429-1437 (2002) (in Russian).

[24]Bajt, S., Stearns, D. G. and Kearney, P. A., "Investigation of the amorphous-to-crystalline transition in Mo-Si multilayers," J. Appl. Phys. 90, 1017-1025 (2001).

[25] Andreev, S. S., Gaponov, S. V., Gusev, S. A., Haidl, M. N., Kluenkov, E. B., Prokhorov, K. A., Polushkin, N. I., Sadova, E. N., Salashchenko, N. N., Suslov, L. A., Zuev, S. Yu., "The microstructure and X-ray reflectivity of Mo/Si multilayers," Thin Solid Films 415, 123-132 (2002).

[26] Vernon, Stephen P. ,Stearns, Daniel G. and Rosen, Robert S., "Ion-assisted sputter deposition of molybdenumsilicon multilayers," Appl. Opt. 32(34), 6969-6974 (1993).

[27] Patelli, A., Rigato, V., Salmaso, G., Carvalho, N. J. M., De Hosson, J. Th. M., Bontempi, E. and Depero, L.E., "Ion bombardment effects on nucleation of sputtered Mo nano-crystals in Mo/B4C/Si multilayers," Surface \& Coat. Technol. 201,143-147 (2006).

[28]Zubarev, E. N., Kondratenko, V.V., Sevryukova, V.A., Yulin, S.A., Feigl, T. and Kaiser, N., "The structure of Mo/Si multilayers prepared in the conditions of ionic assistance," Appl. Phys. A90, 705-710 (2008).

[29] Jankowski, A. F., "On eliminating deposition-induced amorphization of interfaces in refractory metal multilayer systems," Thin Solid Films 220, 166-17I (1992).

[30] Yamaguchi, T., Ikuta, H., Tomofuji, T., Yanagi, Y., Itoh, Y., Oka, T. and Mizutani, U., "Reflective properties of $\mathrm{Mo} / \mathrm{Si}$ multilayer for EUV lithography deposited by the magnetron sputtering device with superconducting bulk magnets," Physica C 468, 2170-2173 (2008).

[31] Niibe, Masahito, Miyafuji, Atsushi, Kinoshita, Hiroo, Watanabe, Takeo, Inoue, Shozo and Koterazawa, Keiji, "Fabrication of an aspherical mirror for Extreme Ultraviolet Lithography (EUVL) optics," Proc. SPIE 3447,32-39 (1998).

[32] Hosokawa, Nobuyuki, Watanabe, Takeo, Sakaya, Noriyuki, Shoki, Tsutomu, Hamamoto, Kazuhiro and Kinoshita, Hiroo, "Development of beam splitter using multilayer membrane for extreme ultraviolet phase-shift microscope," Jap. J. Appl. Phys. 44, 5540-5543 (2005).

[33] Minaichev, V. E., Odinokov, V.V. and Tyufaev, G.P., "Magnetron sputtering equipment, ser.7," Review of electronic engineering, 8(659), 57 (1979) (in Russian).

[34] Ruppert, A. F., Persans, P. D., Hughes, G. J. Liang, K. S., Abeles, B. and Lanford, W., "Density of ultrathin amorphous silicon and germanium sublayers in periodic amorphous multilayers," Phys. Rev. B 44, 11381-11385 (1991).

[35] Gautier, J., Delmotte, F., Bridou, F., Ravet, M. F., Varniere, F., Roulliay, M., Jerome, A. and Vickridge, I., "Characterization and optimization of magnetron sputtered Sc/Si multilayers for extreme ultraviolet optics," Appl. Phys. A88, 719-725 (2007).

[36] Hoffman, D. W. and Thornton, J. A., "Internal stresses in Cr, Mo, Ta and Pt films deposited by sputtering from a planar magnetron source," J. Vac. Sci. Technol. 20, 355-358 (1982).

[37]Zubarev, E. N., Pershyn, Y.P., Kondratenko, V.V., "Unpublished work" (2011).

[38] Niibe, Masahito, Hayashida, Masami, Tizuka, Takashi, Miyake, Akira, Watanabe, Yutaka, Takahashi, Rie and Fukuda, Yasuaki, "Suppression of columnar-structure formation in Mo-Si layered synthetic microstructures," Proc. SPIE 1343, 2-13 (1990).

[39] Heidemann, B., Tappe, T., Schmiedeskamp, B. and Heinzmann, U., "Interlayer composition and interface stability in Mo/Si multilayers studied with high-resolution RBS,” Appl. Surf. Sci. 78, 133-140 (1994). 
[40] Yakshin, A. E., Louis, E., Gorts, P. C., Maas, E. L. G. and Bijkerk, F., "Determination of the layered structure in Mo/Si multilayers by grazing incidence X-ray reflectometry," Physica B 283, 143-148 (2000).

[41] Nedelcu, I., van de Kruijs, R. W. E., Yakshin, A. E. and Bijkerk, F. "Temperature-dependent nanocrystal formation in Mo/Si multilayers," Phys. Rev. B 76, 245404 (2007).

[42] Maury, H., Jonnard, P., André, J.-M., Gautier, J., Roulliay, M., Bridou, F., Delmotte, F., Ravet, M.-F., Jérome, A. and Holliger, P. "Non-destructive X-ray study of the interphases in $\mathrm{Mo} / \mathrm{Si}$ and $\mathrm{Mo} / \mathrm{B}_{4} \mathrm{C} / \mathrm{Si} / \mathrm{B}_{4} \mathrm{C}$ multilayers," Thin Solid Films 514, 278-286 (2006).

[43]Zubarev, E. N., Mel'nik, V. P., Kondratenko, V. V., Pen'kov, A. V., Ponomarenko, A. G., Romanyuk, B. N. and Sevryukova, V. A., "Intermixing processes in $\mathrm{Mo} / \mathrm{Si}$ multilayer periodic compositions irradiated by $\mathrm{Ar}+$ ions," Metallofizika i noveishie tehnologii 29(12), 1555-1570 (2007) (in Russian).

[44] Underwood, J. H., Gullikson, E. M. and Nguyen, Khanh, "Tarnishing of Mo/Si multilayer x-ray mirrors," Appl. Opt. 32, 6985-6990 (1993). 